\title{
Peak Coalescence, Spontaneous Loss of Coherence, and Quantification of the Relative Abundances of Two Species in the Plasma Regime: Particle-In-Cell Modeling of Fourier Transform Ion Cyclotron Resonance Mass Spectrometry
}

\author{
M. Takeshi Nakata, Grant W. Hart, and Bryan G. Peterson \\ Department of Physics and Astronomy, Brigham Young University, Provo, Utah, USA
}

Fourier transform ion cyclotron resonance mass spectrometry (FTICR-MS) is often limited by space-charge effects. Previously, particle-in-cell (PIC) simulations have been used to understand these effects on FTICR-MS signals. However, none have extended fully into the space-charge dominated (plasma) regime. We use a two-dimensional (2-D) electrostatic PIC code, which facilitates work at very high number densities at modest computational cost to study FTICR-MS in the plasma regime. In our simulation, we have observed peak coalescence and the rapid loss of signal coherence, two common experimental problems. This demonstrates that a 2-D model can simulate these effects. The 2-D code can handle a larger numbers of particles and finer spatial resolution than can currently be addressed by 3-D models. The PIC method naturally takes into account image charge and space charge effects in trapped-ion mass spectrometry. We found we can quantify the relative abundances of two closely spaced (such as ${ }^{7} \mathrm{Be}^{+}$and ${ }^{7} \mathrm{Li}^{+}$) species in the plasma regime even when their peaks have coalesced. We find that the frequency of the coalesced peak shifts linearly according to the relative abundances of these species. Space charge also affects more widely spaced lines. Singlyionized ${ }^{7} \mathrm{BeH}$ and ${ }^{7} \mathrm{Li}$ have two separate peaks in the plasma regime. Both the frequency and peak area vary nonlinearly with their relative abundances. Under some conditions, the signal exhibited a rapid loss of coherence. We found that this is due to a high order diocotron instability growing in the ion cloud. (J Am Soc Mass Spectrom 2010, 21, 1712-1719) (c) 2010 American Society for Mass Spectrometry

$\mathrm{F}$ ourier transform ion cyclotron resonance mass spectrometry (FTICR-MS) is often limited by space-charge effects. These effects come from the mutual repulsion of like-charged particles. To improve spectral strength and dynamic range, it is often desirable to work near the space-charge limit where these collective effects become important. The regime where these effects dominate is known as the plasma regime.

A non-neutral plasma is defined as a gas of likecharged particles that exhibits collective behavior. An effective measure of these effects is the Debye length of this collection. The smaller the Debye length compared with the radius of the ion cloud, the more the cloud behaves as a non-neutral plasma, where the collective behavior dominates. The Debye length, $\lambda_{D}$, is the distance over which a collection of charged particles shields an applied electric field. It is defined as follows:

Address reprint requests to G. W. Hart, Department of Physics and Astronomy, Brigham Young University, Provo, Utah 84602, USA. E-mail: grant_hart@byu.edu

$$
\lambda_{D}=\sqrt{\frac{\epsilon_{0} k T}{n e^{2}}}
$$

where $n$ is the particle density, $T$ is the temperature of the ions, $k$ is the Boltzmann constant, $e$ is the charge of the ions, and $\epsilon_{0}$ is the permittivity of free space. Peurrung et al. [1] give a good introduction to the physics of non-neutral plasmas and its relevance to cyclotron resonance mass spectrometry.

FTICR-MS is not well understood in this high number density regime. Some of the initial work to understand this Coulomb interaction was done by Jeffries et al. [2] for weak space-charge effects. Later, Gorshkov et al. [3] considered two species using a line charge model, with both line charges orbiting at the same radius. However, none of these approaches can fully explain what happens in an ion cloud at higher number densities.

In two FTICR-MS experiments, Huang et al. [4] and Naito and Inoue [5] observed that the spectral peaks for similar mass species coalesced at high densities. Naito 
and Inoue [5, 6] modeled this phenomenon with two singly-charged particles confined in a plane perpendicular to a uniform magnetic field. They calculated that one of the mode frequencies of this four degrees-of-freedom system is at the molecular-number weighted average of the two individual cyclotron frequencies. They speculate that this frequency is the frequency of the coalesced peak.

Using a numerical simulation of two cylindrical ion clouds, Mitchell and Smith [7] observed that the frequency after phase locking was almost, but not quite, the weighted average of their unperturbed cyclotron frequencies. In a subsequent paper [8], they observed that there was a relative ion abundance dependence on the single collective cyclotron frequency of cylindrical ion clouds. In this paper, they studied extensively the various parameters which affect the phase locking of ion clouds.

In a pure electron plasma, Gould and LaPointe [9] observed that the $m=1$ cyclotron mode (which is the oscillation that produces the FTICR-MS signal in a non-neutral plasma) had a frequency equal to the cyclotron frequency downshifted by the diocotron frequency. The diocotron frequency is associated with the $\mathbf{E} \times \mathbf{B}$ drift, which also causes the entire charge column to rotate. The diocotron mode is due to the radial electric fields from the image charges on the confining wall. In a non-neutral Mg ion plasma, Sarid et al. [10] also observed a shift in these cyclotron modes. They used Davidson's [11] multispecies cold plasma theory to explain their observed frequency shifts for the major species of ${ }^{24} \mathrm{Mg}^{+}$and ${ }^{24} \mathrm{Mg}^{2+}$. However, they could not explain the shifts for the minor species of ${ }^{25} \mathrm{Mg}^{+},{ }^{26} \mathrm{Mg}^{+}$, and impurities of $\mathrm{H}_{2} \mathrm{O}^{+}$and $\mathrm{H}_{3} \mathrm{O}^{+}$in their system.

To understand trapped-ion mass spectrometry in high space-charge conditions, but not fully into the plasma regime, Mitchell and Smith [12] used a 2-D particle-in-cell (PIC) simulation to demonstrate that image charge detection of a single species ion cloud could be reliably done to make realistic predictions. Later, Mitchell [13] simulated the coalescence phenomenon in a 3-D PIC code. He noticed that the frequency at phase locking for two equal amounts of two species was an average of the two shifted cyclotron frequencies. The shift of cyclotron frequencies was due to the image charge interaction between the phase-locked ion cloud and the conducting wall. He used the Gorshkov et al. [3] calculation of the image charge frequency shift for a line charge inside a grounded cylindrical trap. This result Mitchell thought was fortuitous because his simulation was in a cubic trap and not in an infinitely long cylindrical trap. Nikolaev et al. [14] used a 3-D PIC code to understand the coherence of a single-species ion cloud when its cyclotron motion is excited. They also studied the peak coalescence of two species with similar mass-to-charge ratios and the complex dynamics of three species with similar mass-to-charge ratios.

In this paper, we use a 2-D PIC simulation to study the FTICR-MS signal fully into the plasma regime. Our application of this tool was to see if we could quantify the relative abundances of ${ }^{7} \mathrm{Be}^{+}$and ${ }^{7} \mathrm{Li}^{+}$in the plasma regime in a cylindrical trap. We also studied ${ }^{7} \mathrm{BeH}^{+}$and ${ }^{7} \mathrm{Li}^{+}$. Our motivation for this measurement is to study the radioactive decay rate of singly-ionized beryllium-7 $\left({ }^{7} \mathrm{Be}^{+}\right)$ions. ${ }^{7} \mathrm{Be}$ decays only by electron capture to lithium-7 $\left({ }^{7} \mathrm{Li}\right)$, thereby making its half-life depend on its electronic state $[15,16]$. Getting adequate decay statistics requires about $10^{9}{ }^{7} \mathrm{Be}$ ions, which in our cylindrical trap corresponds to a density of $10^{7}$ particles per $\mathrm{cm}^{-3}$. Under these conditions the radius of the ion cloud is about 50 Debye lengths at room temperature. In contrast, the densest ion clouds simulated by Mitchell as well as Nikolaev et al. had a radius of about two Debye lengths at room temperature, a value more than an order of magnitude smaller than our conditions.

To model the dynamics correctly it is necessary for the computational grid to resolve the Debye length. Since we have more Debye lengths per radius, we require a finer computational grid than has been used in past simulations. In particular, we used a $426 \times 426$ grid. This compares to Nikolaev's finest grid of $128 \times 128 \times$ 128. The computational cost of extending the much finer grid to 3-D is unacceptably high. Staying in 2-D also allows us to work with more simulation particles, reducing the shot noise in the results. As a result, this code can extend previous simulation studies fully into the plasma regime where high-density effects can be probed. We can model plasmas with densities up to the Brillouin density limit $\left(n_{B}=\epsilon_{0} B^{2} / 2 m\right)$, which is the maximum number density a collection of ions can have and still be confined in a trap like this [17].

\section{Methods}

The code used in this paper is a 2-D, collisionless, electrostatic PIC code. It moves the particles using a Boris [18]-Buneman [19] mover, builds a density grid using bilinear interpolation, and solves Poisson's equation using a direct banded-matrix solver [20]. In this solver the finite-differenced equations for Poisson's equation are written as a banded matrix. The solution is obtained by inverting this banded matrix by LU decomposition [21]. For more specific details about how a PIC code works, we refer to Birdsall and Langdon's [22] and Hockney and Eastwood's [23] texts.

Our PIC code specifically uses a Cartesian computational grid with a circular boundary to avoid the difficulties at the origin in polar coordinates. Since the Cartesian grid does not fit the circular boundary, it is necessary to use short-legged operators at the grid points adjacent to the boundary [24, 25]. We used a $426 \times 426$ grid to resolve our plasma Debye length of $377 \mu \mathrm{m}$ and a $30 \mathrm{~ns}$ time step for an accuracy of four significant figures in frequency. This $30 \mathrm{~ns}$ time step allows us to clearly resolve the cyclotron frequencies of ${ }^{7} \mathrm{Be}^{+}$and ${ }^{7} \mathrm{Li}^{+}$, which differ only by $124 \mathrm{~Hz}$ and have a runtime of $2.5 \mathrm{wk}$ for our longest simulation runs.

We simulate a two-species plasma in a cylindrical trap with both species being positive ions. The confined 
plasma has a radius of $2 \mathrm{~cm}$ at room temperature with a flattop radial profile. The positions and velocities of each particle are initialized by a thermal Maxwellian distribution with a constant radial profile (which can be changed to fit the experiment). The initial velocities of each particle also include an initial equilibrium velocity profile from $\mathbf{E} \times \mathbf{B}$ drift.

The conducting wall is divided into four sectors and has a radius of $4 \mathrm{~cm}$. The axial magnetic field is $0.43 \mathrm{~T}$. In making the simulation 2-D, we ignore the axial velocity and the effects of the axial trapping oscillation that exists in an experimental trap. We also assume that the particles in our plasma do not collide in a manner that affects their cyclotron frequencies. The collision time between ions in our system is about $2 \mathrm{~ms}$ and the period for our cyclotron motion is about $1 \mu \mathrm{s}$. The ionneutral collision time at a pressure of $10^{-9}$ torr is of the order of days, so we do not need to be concerned about ion-neutral collisions. We also assume that our magnetic field is constant and does not change with time.

In our simulation, we applied the following waveform on the boundary for broadband excitation:

$$
f(t)=a t_{d r} \frac{\sin \left(\omega_{1} \tau\right)-\sin \left(\omega_{2} \tau\right)}{\pi \tau}
$$

where $a$ is the spectral amplitude, $t_{d r}$ is the driving time, $\omega_{1}$ is the start angular frequency, $\omega_{2}$ is the end angular frequency, and $\tau=t-.5 t_{d r}$. The Fourier transform of this waveform results in a rectangular function at given amplitude $a$ and frequencies $\left[\omega_{1} \cdot \omega_{2}\right]$ with Gibb's oscillations on each edge. We have also used SWIFT excitation [26-28] on the boundary with the same spectrum and it gives the same results.

We detect the induced charge on a wall sector by integrating the surface charge density for a given arc sector on the boundary. This surface charge density is proportional to the radial component of the electric field. The electric field on the boundary is calculated by quadratically extrapolating the electric potential at the boundary and two nearest interior grid points in the simulation. After each simulation run, we calculate the difference of the charge induced on two opposing sectors on the wall. We multiply this by a Hann window function and fastFourier transform this signal in MATLAB to obtain the desired spectrum.

To find the spectral peak frequency, we use the three data points of highest intensity at the peak to fit each spectral peak. As suggested by Goodner et al. [29], we fit each peak with a Comisarow polynomial of the form $y=\left(a x^{2}+b x+c\right)^{n}$ where $n=5.5$ for a Hann window. To find the area under the spectral peak, we integrate using the trapezoidal method. For more specific details about our PIC code, we refer to Nakata's dissertation [30].

\section{Results and Discussion}

\section{${ }^{7} \mathrm{Be}^{+}$and ${ }^{7} \mathrm{Li}^{+}$Coalescence Study}

We first look at the coalescence of the spectral peaks of ${ }^{7} \mathrm{Be}^{+}$and ${ }^{7} \mathrm{Li}^{+}$as the central densities are varied from $10^{3}$ to $10^{7} \mathrm{~cm}^{-3}$. This is done for equal abundances of each species. Note that the ideal cyclotron frequency for ${ }^{7} \mathrm{Be}^{+}$is $941.028 \mathrm{kHz}$ and for ${ }^{7} \mathrm{Li}^{+}$is $941.152 \mathrm{kHz}$. Figure 1 illus-
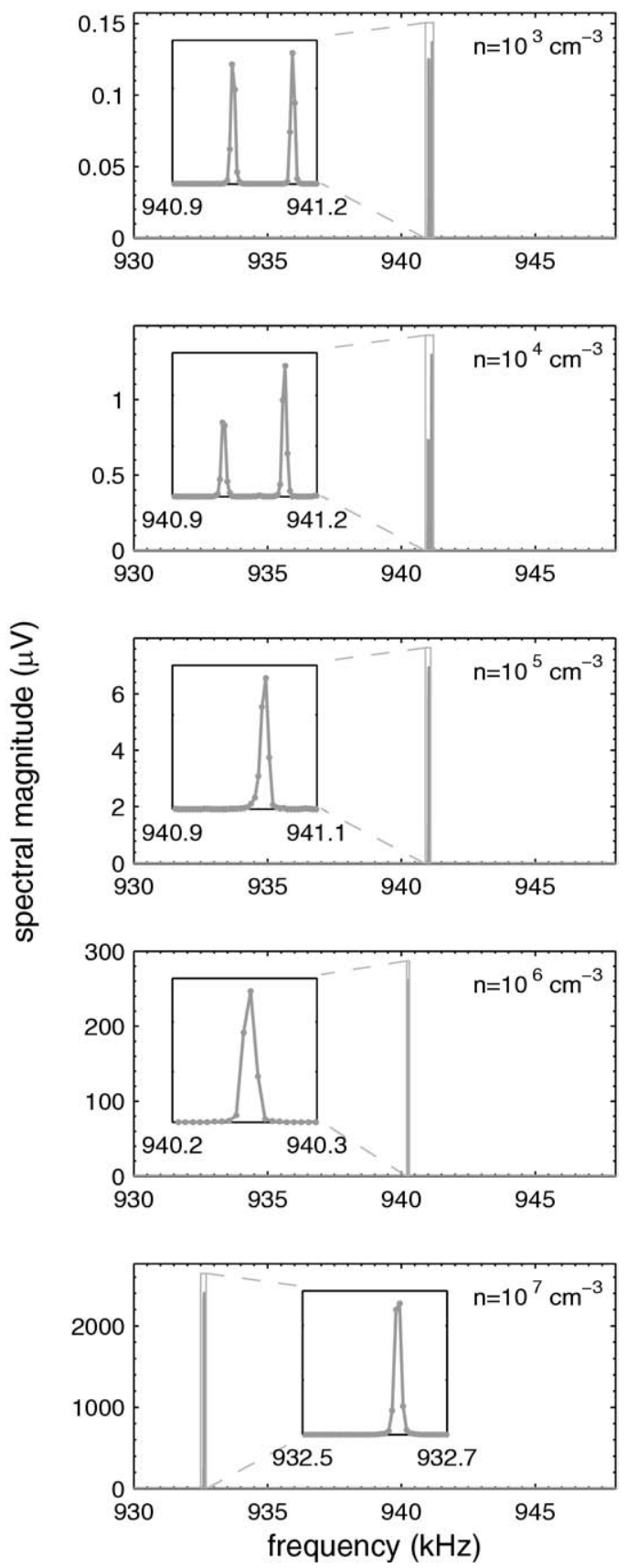

Figure 1. Coalescence of ${ }^{7} \mathrm{Be}^{+}$and ${ }^{7} \mathrm{Li}^{+}$spectral peaks as density increases. This was done for equal abundances of each species. Their relative cyclotron motions were excited by a broadband pulse covering different frequencies for each density at $2.5 \mathrm{~V}$ for $0.1 \mathrm{~ms}$. The signal was observed for $200 \mathrm{~ms}$. The two separate peaks coalesce into one, which shifts in frequency. The density is shown on the top right corner of the graphs. 




Figure 2. Analysis of how the coalesced peak frequency for ${ }^{7} \mathrm{Be}^{+}$ and ${ }^{7} \mathrm{Li}^{+}$ions at a density of $10^{7} \mathrm{~cm}^{-3}$ varies with the fraction of ${ }^{7} \mathrm{Li}^{+}$. The variation is linear in ${ }^{7} \mathrm{Li}^{+}$fraction. The fitted equation is written in the top left corner. The two species were excited by a broadband pulse covering 931 to $933 \mathrm{kHz}$ at $2.5 \mathrm{~V}$ for $0.1 \mathrm{~ms}$. Thereafter, the signal is observed for $200 \mathrm{~ms}$.

trates the changes that occur in the spectrum as the central density is increased.

We observe that the two separate peaks for ${ }^{7} \mathrm{Be}^{+}$and ${ }^{7} \mathrm{Li}^{+}$merge into a single, coalesced peak between central densities of $10^{4} \mathrm{~cm}^{-3}$ and $10^{5} \mathrm{~cm}^{-3}$. This spectral peak also downshifts from the ideal cyclotron frequency as central density increases. This shift becomes large above $10^{6} \mathrm{~cm}^{-3}$.

These results are roughly consistent with Mitchell's [13] stability condition (originally derived from Peurrung and Kouzes [31]) and Boldin and Nikolaev's [32] condition for uniformly charged spheres (even though a more accurate model would be two uniformly charged rods). These conditions both predict that for this particular system, the two spectral peaks should be coalesced at a density $10^{5} \mathrm{~cm}^{-3}$ and not coalesced at a density of $10^{4} \mathrm{~cm}^{-3}$. Note that our results are only consistent at the order of magnitude of these conditions.

\section{Quantifying the Abundances of ${ }^{7} \mathrm{Be}^{+}$and ${ }^{7} \mathrm{Li}^{+}$by Frequency Shift}

We studied how the coalesced spectral peak of ${ }^{7} \mathrm{Be}^{+}$and ${ }^{7} \mathrm{Li}^{+}$at a density of $10^{7} \mathrm{~cm}^{-3}$ varies with abundances. We used 1 million simulation particles in our 2-D PIC code. We excited their respective cyclotron motions with a broadband pulse covering $931 \mathrm{kHz}$ to $933 \mathrm{kHz}$ at $2.5 \mathrm{~V}$ for $0.1 \mathrm{~ms}$. After this excitation, we observed the induced wall signal for $200 \mathrm{~ms}$, which gives us a frequency resolution of $5 \mathrm{~Hz}$ to resolve the cyclotron frequency difference of about $124 \mathrm{~Hz}$.

We observed how the coalesced spectral peak shifts with the relative abundances of the two species. The merged peak moves from the ${ }^{7} \mathrm{Be}^{+}$frequency to the ${ }^{7} \mathrm{Li}^{+}$ frequency as the ratio of ${ }^{7} \mathrm{Li}^{+}$to ${ }^{7} \mathrm{Be}^{+}$increases. The spectral magnitude remains the same for each ratio when we apply the same excitation to our system. Figure 2 shows that the coalesced peak frequency shifts linearly as a function of the ${ }^{7} \mathrm{Li}^{+}$fraction at a total density (held constant) of $10^{7} \mathrm{~cm}^{-3}$. The coalesced peak frequency is the weighted average of the individual cyclotron frequencies of ${ }^{7} \mathrm{Be}^{+}$and ${ }^{7} \mathrm{Li}^{+}$downshifted by $8.458 \mathrm{kHz}$.

For an infinitely-long cylindrical plasma, Levy [33] calculated the diocotron frequency as follows:

$$
\omega_{d}=\frac{\lambda}{2 \pi \epsilon_{0} R_{w}^{2} B_{z}}
$$

where $\omega_{d}$ is the diocotron angular frequency, $\lambda$ is the charge per unit length, $R_{w}$ is the radius to the wall, and $B_{z}$ is the axial magnetic field strength. This diocotron frequency shifts with large amplitude [34] and finite length [35]. For this case, the measured diocotron frequency from the FTICR-MS spectrum is $8.500 \mathrm{kHz}$ which is a $42 \mathrm{~Hz}$ difference from the coalesced peak's observed shift. This difference is comparable to the systematic numerical shift in the frequency due to the finite time step. Based on our simulations it is reason-

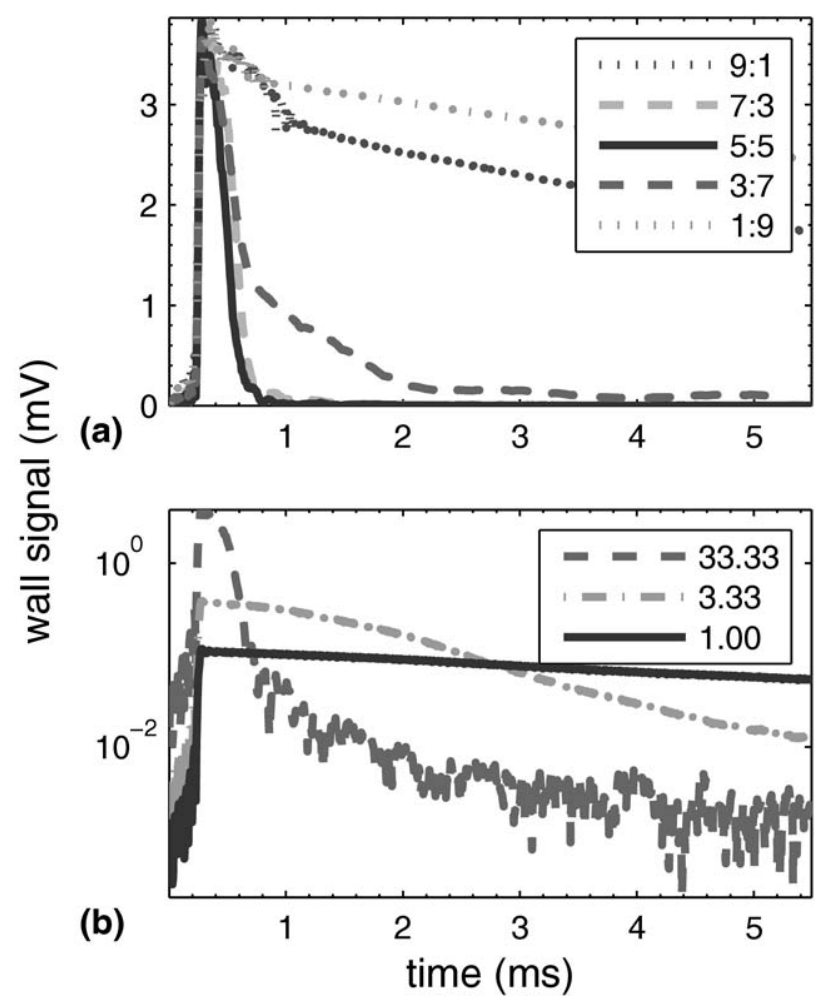

Figure 3. (a) The envelopes of amplitudes as the ratio of ${ }^{7} \mathrm{BeH}^{+}$ to ${ }^{7} \mathrm{Li}^{+}$in the plasma is varied at a driving amplitude of $33.33 \mathrm{mV}$. The values on the top right are ratios of ${ }^{7} \mathrm{BeH}^{+}$to ${ }^{7} \mathrm{Li}^{+}$. Notice that the transient decays fastest at equal ratios of the two species; (b) is the envelopes of amplitudes of ${ }^{7} \mathrm{BeH}^{+}$and ${ }^{7} \mathrm{Li}^{+}$as the driving amplitude is varied for equal amounts of each. The $y$-axis is on a $\log$ scale. The values on the top right are driving amplitudes in units of $\mathrm{mV}$. All of these amplitudes are below the ejection limit. Notice that the transient decays fastest at the largest driving amplitude of $33.33 \mathrm{mV}$. For both graphs, the plasma has a total central density of $10^{7} \mathrm{~cm}^{-3}$ and the cyclotron motion is excited from $t=0$ to $0.5 \mathrm{~ms}$. 
able to say that the individual cyclotron frequencies at this high density are downshifted by the diocotron frequency.

Indeed, Mitchell [13] observed a frequency shift for two ion clouds of similar masses and with equal numbers at high density, but with only two Debye lengths in the plasma, in a 3-D PIC code. He attributed this shift to the image charge interaction acting on the phase locked ion cloud. Further, Mitchell and Smith [8] observed that the coalesced peak frequency showed a similar (but not explicitly specified) dependence on the relative abundance. Our results extend these observations into the plasma regime. The individual cyclotron frequencies are shifted by the diocotron frequency of the plasma. The coalesced peak frequency does depend linearly on the relative abundances of the two species even in the plasma regime.

The simulation suggests how to determine the abundance of a two species plasma experimentally: (1) obtain the diocotron frequency, the coalesced frequency, and a reference frequency from an impurity species that is not coalesced in the spectrum; (2) calculate the magnetic field of the system using the known mass of the impurity species and the diocotron frequency; (3) Add the coalesced frequency to the diocotron frequency to obtain the corrected coalesced frequency; (4) using this corrected frequency and the cyclotron frequencies of the two species, determine the relative abundance of each species.

\section{${ }^{7} \mathrm{BeH}^{+}$and ${ }^{7} \mathrm{Li}^{+}$Time Signal Decays}

We also studied ${ }^{7} \mathrm{BeH}^{+}$and ${ }^{7} \mathrm{Li}^{+}$because at least a portion of the singly ionized ${ }^{7} \mathrm{Be}^{+}$in our vacuum system will bond with residual hydrogen to form ${ }^{7} \mathrm{BeH}^{+}$[36]. This case is experimentally less challenging compared to the ${ }^{7} \mathrm{Be}^{+}$and ${ }^{7} \mathrm{Li}^{+}$case because the mass difference is larger. As a result, the individual peaks will not coalesce. However, the simulation shows that coupling effects are still important.

We find that the time signal driven at an amplitude of $33 \mathrm{mV}$ for equal amounts of ${ }^{7} \mathrm{BeH}^{+}$and ${ }^{7} \mathrm{Li}^{+}$at a density of $10^{7} \mathrm{~cm}^{-3}$ ions quickly decays. The top graph in Figure 3 shows this decay effect. At other ratios of ${ }^{7} \mathrm{BeH}^{+}$to ${ }^{7} \mathrm{Li}^{+}$the transients decay more slowly. We also simulated this signal at a density of $10^{3} \mathrm{~cm}^{-3}$ and find little transient decay, which leads us to conclude that this is a plasma effect.

This effect also depends on the strengths of the excitation amplitude. This is illustrated for a plasma consisting of equal amounts of ${ }^{7} \mathrm{BeH}^{+}$and ${ }^{7} \mathrm{Li}^{+}$in the bottom graph in Figure 3. The decay rate lessens as the excitation spectral amplitude drops from $33 \mathrm{mV}$ to 1 $\mathrm{mV}$. At $33 \mathrm{mV}$, the decay occurs within less than $0.5 \mathrm{~ms}$.



(a)

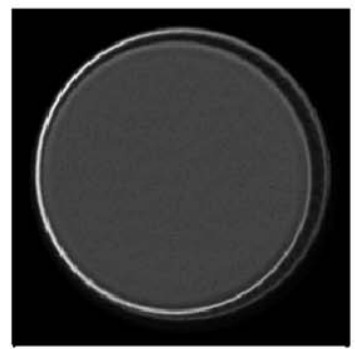

(b) $\mathrm{t}_{1}=0.317 \mathrm{~ms}$

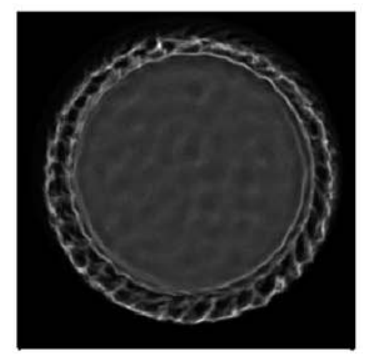

$\mathrm{t}_{2}=0.389 \mathrm{~ms}$

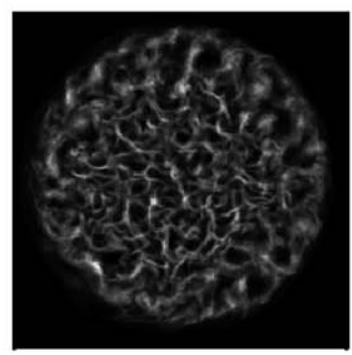

$\mathrm{t}_{3}=0.498 \mathrm{~ms}$

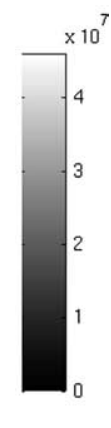

Figure 4. (a) The induced wall signal of ${ }^{7} \mathrm{BeH}^{+}$and ${ }^{7} \mathrm{Li}^{+}$ion plasma at a density of $10^{7} \mathrm{~cm}^{-3}$. Equal amounts of each species were excited from $t=0$ to $0.5 \mathrm{~ms}$ with two broadband pulses of amplitude of $33 \mathrm{mV}$ at $785 \mathrm{kHz}$ to $815 \mathrm{kHz}$ and at $905 \mathrm{kHz}$ to $935 \mathrm{kHz}$. The three black lines indicate the times of each density snapshot; (b) shows the total density plot of the plasma at the indicated times. The wall (not shown here) is located at twice the plasma radius. The color bar on the bottom right measures the density in units of $\mathrm{cm}^{-3}$. Notice how the initial hollow circular region evolves to destroy the coherence of the cyclotron oscillations. 
By $3.33 \mathrm{mV}$, it has slowed down to about $5 \mathrm{~ms}$. At $1 \mathrm{mV}$, it decays at a still slower rate.

Density plots from the simulation show the cause of this effect. The bottom three frames in Figure 4 illustrate how the total density of the plasma evolves with time. Frame $t_{1}$ shows that a hollow circular region forms between the bulk of the plasma and a tail that streams from the outer edge, early in the driving period. This region is unstable and results in a high order (many spatial periods around the circumference) diocotron instability $[33,37]$ as shown in frame $t_{2}$. By frame $t_{3}$, this instability has non-linearly grown to destroy any coherence of the fundamental cyclotron oscillations.

Aizikov et al. [38] describe this phenomenon as a spontaneous loss of coherence catastrophe (SLCC). They noted that Nikolaev et al. [14] also observed this phenomenon in their simulation. However, in Nikolaev's simulation, this decoherence phenomenon occurred for a single mass-to-charge species, which exclusively occurred in three dimensions. Nonetheless, both their effect and this 2-D, two-species effect can contribute to the decoherence of the wall time signal. Understanding both of these mechanisms is important to avoid this coherence catastrophe.

\section{Quantifying the Abundances of ${ }^{7} \mathrm{BeH}^{+}$and ${ }^{7} \mathrm{Li}^{+}$}

To quantitatively study how the relative abundances of ${ }^{7} \mathrm{BeH}^{+}$and ${ }^{7} \mathrm{Li}^{+}$at a density of $10^{7} \mathrm{~cm}^{-3}$ are related in FTICR-MS, we used 10 million simulation particles in our code. We excited the ${ }^{7} \mathrm{BeH}^{+}$cyclotron motion with a broadband pulse at $785 \mathrm{kHz}$ to $815 \mathrm{kHz}$ and the ${ }^{7} \mathrm{Li}^{+}$ cyclotron motion with a broadband pulse at $905 \mathrm{kHz}$ to $935 \mathrm{kHz}$, both driven at $1 \mathrm{mV}$ for $0.5 \mathrm{~ms}$. After the excitation was applied, we observed the induced wall signal for $5 \mathrm{~ms}$. We kept the total density constant as we varied the relative abundances of the two species.

We observed that the ${ }^{7} \mathrm{BeH}^{+}$and ${ }^{7} \mathrm{Li}^{+}$spectral peaks remain separate and their respective spectral peak frequencies shift with relative abundance. As the abundance of the more massive ${ }^{7} \mathrm{BeH}^{+}$ions decrease, the two spectral peaks move farther apart and both move away from the pure ${ }^{7} \mathrm{BeH}^{+}$peak. Meanwhile, the ${ }^{7} \mathrm{Li}^{+}$ spectral peak moves toward its pure spectral peak. Another interesting aspect to note is how the spectral peak height also changes nonlinearly with relative abundances.

To better understand the spectral peak shifts with abundances of ${ }^{7} \mathrm{BeH}^{+}$and ${ }^{7} \mathrm{Li}^{+}$at high density, we applied Davidson's [11] multispecies cold plasma theory to a two species plasma. Figure 5 compares the results of this theory with our simulation results for the peak frequency as a function of ${ }^{7} \mathrm{Li}^{+}$fraction. The agreement is very good; the average percent error is $0.005 \%$ (about 50 $\mathrm{Hz}$ ). This theory works just as well for a central number density of $n_{0}=10^{6} \mathrm{~cm}^{-3}$.

We can also determine relative abundances by analyzing the area under the peak. Figure 6 illustrates how the area under the peaks varies with relative abun- dance. As can be seen from the graph, the trends are not linear, but by calibrating these trends with the code we can use this as an independent measure of abundances.

In contrast to the ${ }^{7} \mathrm{Be}^{+}$and ${ }^{7} \mathrm{Li}^{+}$case, we have two independent measurements of the abundances, by using the spectral peak shifts and the changes in area. This may be sufficiently advantageous experimentally that we may want to encourage the formation of ${ }^{7} \mathrm{BeH}^{+}$by adding hydrogen to our trap to bond with the initial ${ }^{7} \mathrm{Be}^{+}$ion plasma.

\section{Conclusions}

A 2-D PIC simulation is extremely useful to understand FTICR-MS in the plasma regime. For example, we were able to observe the abundance dependence of the coalescence of the spectral peaks of two species with similar mass-to-charge ratios. We find that the variation of the frequency is linear with respect to the relative abundances. These results are consistent with the previous theoretical work and fully extend them into the plasma regime.

There are other space-charge related frequency shifts as well. For the well-separated peaks of ${ }^{7} \mathrm{BeH}^{+}$and ${ }^{7} \mathrm{Li}^{+}$, the frequency variation is not linear, but we can

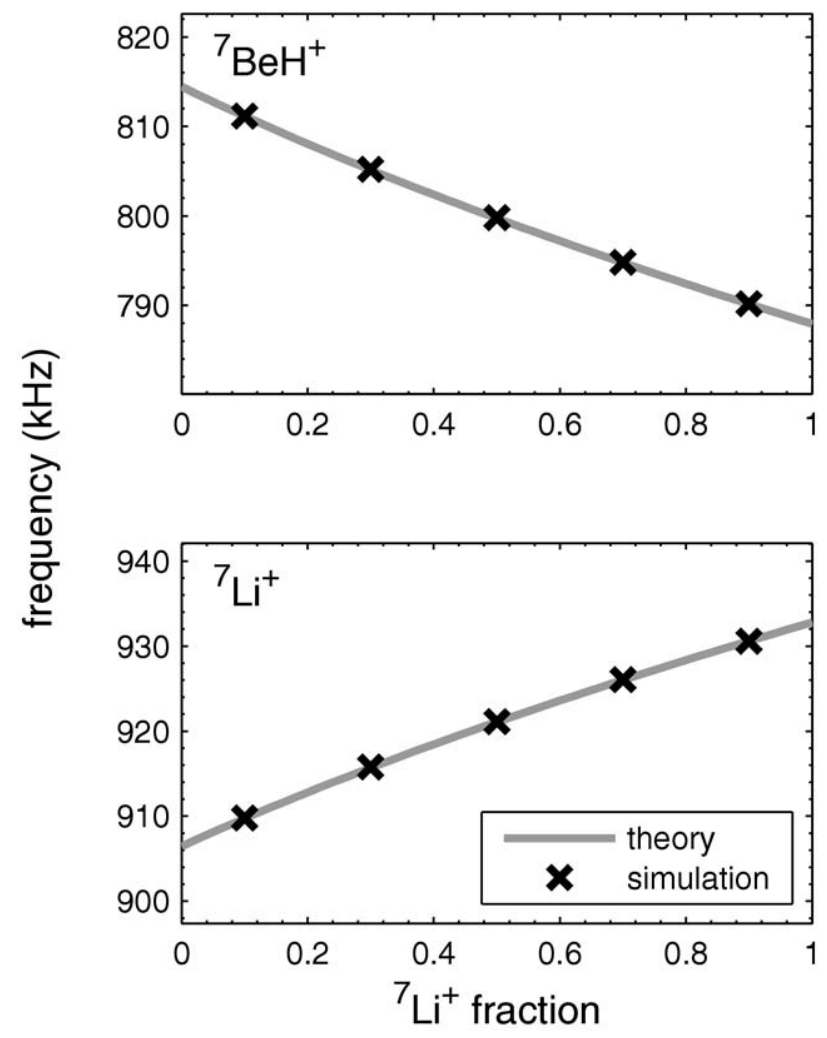

Figure 5. Analysis of how the FTICR-MS spectral peaks for ${ }^{7} \mathrm{BeH}^{+}$and ${ }^{7} \mathrm{Li}^{+}$vary with fraction of ${ }^{7} \mathrm{Li}^{+}$at a central density of $10^{7} \mathrm{~cm}^{-3}$. The species are labeled on the top left corner of each graph. It fits well with Davidson's [11] multispecies cold plasma theory. The average percent error between the theory and simulation is $0.005 \%$. The pure observed frequencies for ${ }^{7} \mathrm{BeH}^{+}$is $814.442 \mathrm{kHz}$ and ${ }^{7} \mathrm{Li}^{+}$is $932.778 \mathrm{kHz}$. 


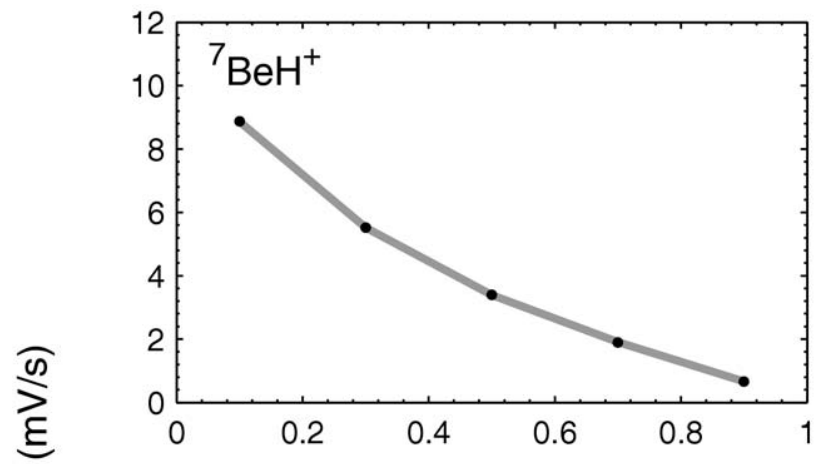

ฮั

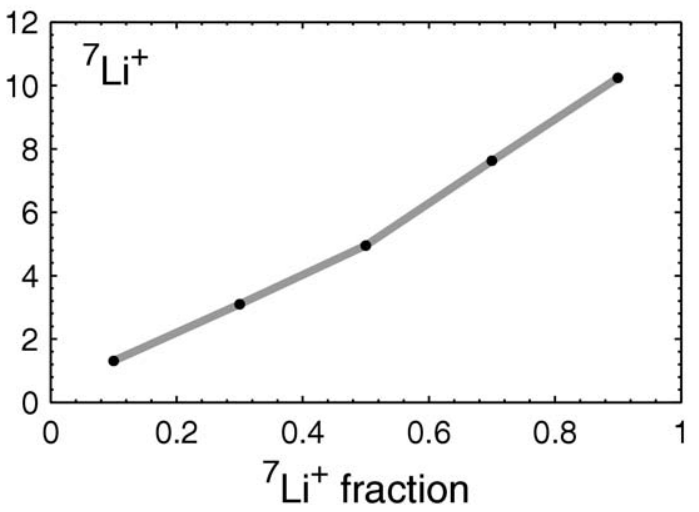

Figure 6. Analysis of how the area under the FTICR-MS spectral peak for ${ }^{7} \mathrm{BeH}^{+}$and ${ }^{7} \mathrm{Li}^{+}$varies with fraction of ${ }^{7} \mathrm{Li}^{+}$. The species is shown on the top left corner of each graph. The dots were connected to illustrate how the trends are not linear. We use a trapezoidal method to calculate the area. Both analyses are for a plasma with a density of $10^{7} \mathrm{~cm}^{-3}$.

use Davidson's [11] theory to correctly predict the frequency shifts. In addition, the peak area can be used as an independent measure of abundances in the plasma regime. This code has also clarified that the loss of coherence that occurs in the transients for the ${ }^{7} \mathrm{BeH}^{+}$ and ${ }^{7} \mathrm{Li}^{+}$at high excitation occurs because of a diocotron instability that develops before the excitation is completed.

A PIC simulation is a tool to understand how to obtain the best possible signal from the FTICRMS technique. Potential future studies can include the study of the preservation of coherence technique known as electron-promoted ion coherence (EPIC) [39, 40], and quantifying the abundances of more complex systems. It could also be used to understand Sarid et al. [10] $\mathrm{Mg}$ ion plasma results and resolve why the theory does not predict the shifts for the minor species. It can also study other more complicated peak coalescence systems such as ${ }^{7} \mathrm{BeH}^{+},{ }^{7} \mathrm{Be}^{+}$, and ${ }^{7} \mathrm{Li}^{+}$.

\section{Acknowledgments}

The authors gratefully acknowledge the College of Physical and Mathematical Science of Brigham Young University and Copley funding for financial support. We also acknowledge the Fulton Supercomputing Lab and its staff for support and computer resources. M. T. N. acknowledges Professor Ross L. Spencer for his theoretical discussions, Professor David V. Dearden for his insights into FTICR-MS physics, and Professor Eric W. Hirschmann for a fresh pair of eyes in reviewing the manuscript.

\section{References}

1. Peurrung, A.; Kouzes, R.; Barlow, S. The Non-Neutral Plasma: An Introduction to Physics with Relevance to Cyclotron Resonance Mass Spectrometry. Int. J. Mass Spectrom. Ion Processes 1996, 157/158, 39-83.

2. Jeffries, J. B.; Barlow, S. E.; Dunn, G. H. Theory of Space-Charge Shift of Ion Cyclotron Resonance Frequencies. Int. J. Mass Spectrom. Ion Processes $1983,54,169-187$.

3. Gorshkov, M. V.; Marshall, A. G.; Nikolaev, E. N. Analysis and Elimination of Systematic Errors Originating from Coulomb Mutual Interaction and Image Charge in Fourier Transform Ion Cyclotron Resonance Precise Mass Difference Measurements. J. Am. Soc. Mass Spectrom. 1993, 4, 855-868.

4. Huang, J.; Tiedemann, P. W.; Land, D. P.; McIver, R. T.; Hemminger J. C. Dynamics of Ion Coupling in an FTMS Ion Trap and Resulting Effects on Mass Spectra, Including Isotope Ratios. Int. J. Mass Spectrom. Ion Processes 1994, 134, 11-21.

5. Naito, Y.; Inoue, M. Peak Confluence Phenomenon in Fourier Transform Ion Cyclotron Resonance Mass Spectrometry. J. Mass Spectrom. Soc. Jpn. 1994, 42, 1-9.

6. Naito, Y.; Inoue, M. Collective Motion of Ions in an Ion Trap for Fourier Transform Ion Cyclotron Resonance Mass Spectrometry. Int. J. Mass Spectrom. Ion Processes 1996, 157/158, 85-96.

7. Mitchell, D. W.; Smith, R. D. Cyclotron motion of two Coulombically Interacting Ion Clouds with Implications to Fourier-Transform Ion Cyclotron Resonance Mass Spectrometry Phys. Rev. E 1995, 52, 4366 4386.

8. Mitchell, D. W.; Smith, R. D. Prediction of a Space Charge Induced Upper Molecular Mass Limit Towards Achieving Unit Mass Resolution in Fourier Transform Ion Cyclotron Resonance Mass Spectrometry. J. Mass Spectrom. 1996, 31, 771-790.

9. Gould, R. W.; LaPointe, M. A. Cyclotron Resonance Phenomena in a Pure Electron Plasma. Phys. Fluids B 1992, 4, 2038-2043.

10. Sarid, E.; Anderegg, F.; Driscoll, C. F. Cyclotron Resonance Phenomena in a Non-Neutral Multispecies Ion Plasma. Phys. Plasmas 1995, 2, 2895-2907.

11. Davidson, R. C. Physics of Non-Neutral Plasma; Addison-Wesley: Redwood City, CA, 1990; p. 258.

12. Mitchell, D. W.; Smith, R. D. Two-Dimensional Many-Particle Simulation of Trapped Ions. Int. J. Mass Spectrom. Ion Processes 1997, 165/166, 271-297.

13. Mitchell, D. W. Realistic Simulation of the Ion Cyclotron Resonance Mass Spectrometer Using a Distributed Three-Dimensional Particle-inCell Code. J. Am. Soc. Mass Spectrom. 1999, 10, 136-152.

14. Nikolaev, E. N.; Heeren, R. M. A.; Popov, A. M.; Pozdneev, A. V.; Chingin, K. S. Realistic Modeling of Ion Cloud Motion in a Fourier Transform Ion Cyclotron Resonance Cell by Use of a Particle-in-Cell Approach. Rapid Commun. Mass Spectrom. 2007, 21, 3527-3546.

15. Segrè, E.; Wiegand, C. E. Experiments on the Effect of Atomic Electrons on the Decay Constant of $\mathrm{Be}^{7}$. Phys. Rev. 1949, 75, 39-43.

16. Das, P.; Ray, A. Terrestrial ${ }^{7}$ Be Decay Rate and ${ }^{8} \mathrm{~B}$ Solar Neutrino Flux. Phys. Rev. C 2005, 71, 025801 1-7.

17. Brillouin, L. A Theorem of Larmor and Its Importance for Electrons in Magnetic Fields. Phys. Rev. 1945, 67, 260-266.

18. Boris, J. P. Relativistic Plasma Simulation-Optimization of a Hybrid Code. Proceedings of the Fourth Conference on the Numerical Simulation of Plasmas; Naval Research Laboratory, Washington, D.C., November 1970; p. 3-67.

19. Buneman, O. Inversion of the Helmholtz (or Laplace-Poisson) Operator for Slab Geometry. J. Comput. Phys. 1973, 12, 124-130.

20. Spencer, R. L.; Mason, G. W. Large Amplitude $\ell=1$ coherent structures in non-neutral plasmas confined in a cylindrical trap. Phys. Fluids $B$ 1993, 5, 1738-1745.

21. Press, W. H.; Teukolsky, S. A.; Vetterling, W. T.; Flannery, B. P. Numerical Recipes in Fortran 77: The Art of Scientific Computing, 2nd ed.; Cambridge University Press: New York, NY, 1992; Sec. 2.3, p. 34-40.

22. Birdsall, C. K.; Langdon, A. B. Plasma Physics via Computer Simulation; McGraw-Hill: New York, NY, 1985; pp 1-504.

23. Hockney, R. W.; Eastwood, J. W. Computer Simulation Using Particles; Taylor and Francis: New York, NY, 1988; pp 1-540.

24. Spencer, R. L. The Effect of Externally Applied Oscillating Electric Fields on the $\ell=1$ and $\ell=2$ Diocotron Modes in Non-Neutral Plasmas. Phys. Fluids B 1990, 2, 2306-2314.

25. Smith, G. D. Numerical Solution of Partial Differential Equations, with Exercises and Worked Solutions; Oxford University Press: New York, NY, 1965; p. 139.

26. Marshall, A. G.; Wang, T.-C. L.; Ricca, T. L. Tailored Excitation for Fourier Transform Ion Cyclotron Mass Spectrometry. J. Am. Chem. Soc. 1985, 107, 7893-7897.

27. Chen, L.; Wang, T.-C. L.; Ricca, T. L.; Marshall, A. G. Phase-Modulated Stored Waveform Inverse Fourier Transform Excitation for Trapped Ion Mass Spectrometry. Anal. Chem. 1987, 59, 449-454. 
28. Guan, S.; McIver, R. T. Jr. Optimal Phase Modulation in Stored Wave Form Inverse Fourier Transform Excitation for Fourier Transform Mass Spectrometry. I. Basic Algorithm. J. Chem. Phys. 1990, 92, 5841-5846.

29. Goodner, K. L.; Milgram, K. E.; Williams, K. R.; Watson, C. H.; Eyler, J. R. Quantitation of Ion Abundances in Fourier Transform Ion Cyclotron Resonance Mass Spectrometry. J. Am. Soc. Mass Spectrom. 1998, 9, 1204-1212.

30. Nakata, M. T. Simulating the FTICR-MS Signal of a Decaying Beryllium-7 Ion Plasma in a $2 D$ Electrostatic PIC Code; Ph.D. Dissertation, Brigham Young University, Provo, UT, 2010.

31. Peurrung, A. J.; Kouzes, R. T. Long-Term Coherence of the Cyclotron Mode in a Trapped Ion Cloud. Phys. Rev. E 1994, 49, 43624368.

32. Boldin, I. A.; Nikolaev, E. N. Theory of Peak Coalescence in Fourier Transform Ion Cyclotron Resonance Mass Spectrometry. Rapid Commun. Mass Spectrom. 2009, 23, 3213-3219.

33. Levy, R. H. Diocotron Instability in a Cylindrical Geometry. Phys. Fluids $1965,8,1288-1295$.
34. Fine, K. S.; Driscoll, C. F.; Malmberg, J. H. Measurements of a Nonlinear Diocotron Mode in Pure Electron Plasmas. Phys. Rev. Lett. 1989, 63, 2232-2235.

35. Fine, K. S.; Driscoll, C. F. The Finite Length Diocotron Mode. Phys. Plasmas 1998, 5, 601-607.

36. Jensen, M. J.; Hasegawa, T.; Bollinger, J. J.; Dubin, D. H. E. Rapid Heating of a Strongly Coupled Plasma near the Solid-Liquid Phase Transition. Phys. Rev. Lett. 2005, 94, 025001 1-4.

37. Davidson, R. C. Physics of Non-Neutral Plasma, Ch. 6; Addison-Wesley: Redwood City, CA, 1990 .

38. Aizikov, K.; Mathur, R.; O'Connor, P. B. The Spontaneous Loss of Coherence Catastrophe in Fourier Transform Ion Cyclotron Resonance Mass Spectrometry. J. Am. Soc. Mass Spectrom. 2009, 20, 247-256.

39. Kaiser, N. K.; Bruce, J. E. Observation of Increased Ion Cyclotron Resonance Signal Duration through Electric Field Perturbations. Anal. Chem. 2005, 77, 5973-5981.

40. Weisbrod, C.; Kaiser, N.; Skulason, G.; Bruce, J. Trapping Ring Electrode Cell: A FTICR Mass Spectrometer Cell for Improved Signal-to-Noise and Resolving Power. Anal. Chem. 2008, 80, 6545-6553. 